\title{
ELEMENTOS METODOLÓGICOS QUE INCIDEN EN LA ENSEÑANZA- APRENDIZAJE DEL ESPAÑOL EN ESTUDIANTES DE PRIMER GRADO, BILWI, RAAN
}

\author{
Petronila Bello Fenly ${ }^{[1]}$ \\ Maritza Gutiérrez Guillermo ${ }^{[2]}$ \\ Argentina García Solórzano ${ }^{[3]}$
}

\section{Resumen}

Este estudio se realizó en la escuela de Educación Especial "Hermana Maureen Courtney", año 2009, Bilwi, RAAN, Nicaragua. Aporta información respecto a los elementos metodológicos que inciden en la enseñanza del español en los estudiantes del primer grado de la Escuela de Educación Especial.

Se utilizó la metodología descriptivo- cualitativa, de corte transversal porque es un estudio sincrónico. Se observó el desarrollo de algunas clases de español, se aplicaron entrevistas abiertas a estudiantes, docentes y subdirectora. Entre los resultados se observó que la docente utilizó una metodología tradicional basada en el método silábico y parte del método Fonético-Analítico-Sintético (FAS), incluyendo la tradicionalidad en la organización del aula, es decir una formación de estudiantes sentados en hileras. Además es poca la utilización de medios y materiales didácticos y casi ausencia en la motivación.

Consideramos de importancia esta investigación para el fortalecimiento de la enseñanza del español en Educación Especial. Por esta razón, se recomienda profundizar en esta temática en futuras investigaciones, lo que ayudará a una mayor sensibilización y mejora de la calidad en la docencia.

Palabras clave: Metodologías del español, Educación Especial, método FAS, medios didácticos.

\footnotetext{
${ }^{[1]}$ Lic. en Ciencias de la Educación con mención en Español, URACCAN. petronilabello @yahoo. es

${ }^{[2]}$ Lic. en Ciencias de la Educación con mención en Español, URACCAN. maritzagutierrez @yahoo. es

${ }^{[3]}$ Secretaria Académica URACCAN Recinto Bilwi. argentina_garcia@yahoo. com
} 


\section{Summary}

This study was conducted at the Special Educational School "Sister Maureen Courtney", during the year 2010, in Bilwi, RAAN, Nicaragua. It provides information regarding the methodological elements that affects the teaching of Spanish in the first grade students of the Special Educational School.

We used a descriptive-qualitative methodology, cross-sectional because it is a synchronic study. We observed the development of some Spanish lessons and interviews were applied to students, teachers and subdirector. Among the results it showed that the teacher used a traditional methodology based on the syllable and Phonics-Analytic-Synthetic methods (FAS), including traditionality in classroom organization, namely, making students to sit in rows. Furthermore there is little use of media and materials and almost no motivation.

We consider this research important for strengthening the teaching of Spanish in Special Education. For this reason it is recommended to deepen this issue in future researches, which will help raise awareness and improve the teaching quality.

Keywords: Spanish methodologies, special education, FAS method, teaching aids.

\section{Introducción}

Este estudio titulado Elementos metodológicos que inciden en la enseñanza- aprendizaje del español en estudiantes de primer grado, Bilwi, RAAN, durante el año escolar 2009. Es vital porque es una escuela única en la región con enfoque a la atención especial en la niñez y jóvenes con discapacidad. Se les atiende en forma especializada ya sea en la asimilación de aprendizajes, retardo mental, problemas de audición y emocionales entre otras cosas.

Ofrece enseñanza primaria, y los estudiantes proceden de distintos centros educativos y comunidades aledañas. La mayoría pertenece a la etnia miskitu, cuya lengua materna es el miskitu, hacia donde se enfoca esta investigación, centrada en primer grado.

La elección del tema obedece precisamente a que como docentes inquieta el hecho de que estos estudiantes presentan serios problemas en el aprendizaje del español, que para ellos es una segunda lengua. Este estudio aportará datos de esa realidad, al destacar a través de las observaciones que llegan al centro con poco conocimiento 
alfabético, traen limitaciones lingüísticas de todo orden, deficiencias en la asimilación, análisis y comprensión de textos en esa lengua.

Este estudio es cualitativo, de corte transversal porque es un estudio sincrónico efectuado en el segundo semestre del 2009. Se analizan documentos cualitativos sobre el rendimiento académico de los últimos años encontrando que en ese grado el estudiantado ha disminuido su calidad escolar en el área de español. Este problema tiene su origen en lo metodológico porque la enseñanza en este centro es netamente monolingüe cuando la lengua materna de la mayoría de los estudiantes es el miskitu.

Durante la observación directa de clases, se analiza el dominio de la metodología aplicada por la docente, la calidad y pertinencia de los medios y materiales didácticos, atención personalizada a los estudiantes, según su procedencia escolar: monolingüe o bilingüe y pertenencia a la etnia miskitu.

Se encontraron deficiencias porque la metodología de la docente es tradicional, no utiliza medios y materiales en el desarrollo de la clase, incrementando las dificultades para el aprendizaje. Esta investigación servirá a las autoridades del centro, al MINED, a los docentes y en especial a los estudiantes para el mejoramiento del proceso de enseñanza del español.

\section{Revisión de la literatura}

Una propuesta metodológica innovadora y los materiales educativos: Para Galdames (2008: 43,48) la utilización de materiales educativos en la sala de clase está basada en una propuesta metodológica innovadora. Esta metodología da especial importancia a la forma en que aprenden los niños y la relación que establecen con ellos y los materiales educativos.

Medios didácticos: Raymond (1978: 7,8) nos define que los medios didácticos proporcionan información práctica, fácilmente comprensible e instrucciones de tipo manual sobre fotografía, elaboración de diapositivas, rótulos, dibujos, tableros de anuncios, montaje de carteles en seco y de muchos otros recursos del oficio.

Guillén y Castro (1998: 95) afirman que los materiales didácticos se enumeran en materiales "tradicionales", que incluyen aquellos usados de "siempre" como son los libros de textos, la pizarra, los mapas, materiales "técnicos" que, según varias generaciones, incluyen todos aquellos que superan al material tradicional con el recurso a los medios audiovisuales, ordenadores, telemática. 
Método de enseñanza: Arredondo (1989: 5,6), define el método de enseñanza como: "el conjunto de momentos y técnicas lógicamente coordinados para dirigir el aprendizaje del estudiante hacia determinados objetivos." Todo método realiza sus operaciones mediante técnicas.

Las técnicas de enseñanza en consecuencia, son también formas de orientación del aprendizaje porque es el camino para llegar a un fin determinado (Ibíd.). También, los métodos de enseñanza descansan sobre las teorías del proceso de aprendizaje y una de las grandes tareas de la pedagogía moderna ha sido estudiar de manera experimental la eficacia de dichos métodos, al mismo tiempo que intenta su formulación teórica.

\section{Interrelación entre las actividades del maestro y los estudiantes en el proceso de enseñanza}

Función del docente: Elkorin (1990: 292) afirma que la función del maestro es la presentación del proceso pedagógico, se parte de la importancia de la actividad del educador. El educador guía el proceso pedagógico, planifica y dirige la actividad de sus estudiantes de manera que se logre la influencia educativa prevista.

\section{Materiales y método}

El presente estudio se realizó en la escuela de Educación Especial "Hna. Maureen Courtney" en la Ciudad de Bilwi, Municipio de Puerto Cabezas, es un estudio descriptivo-cualitativo, de corte transversal porque es una investigación educativa que se realizó en el II semestre del año 2009.

Para su desarrollo se han realizado actividades directas en el campo aplicando las entrevistas individuales a estudiantes, docentes, subdirectora del centro y observaciones de clase como principal forma de recolección de información para el cumplimiento de los objetivos planteados. Se tomó como muestra a 7 varones y 7 mujeres equivalente a un $50 \%$ del total, se entrevistaron a 14 estudiantes de la etnia miskitu, porque ellos son los que presentan problemas en el aprendizaje del español.

\section{Resultados y discusión}

Una vez aplicados los instrumentos de recolección de la información en la Escuela de Educación Especial "Hna. Maureen Courtney", se procesaron llegando a los resultados que a continuación se presentan.

\section{Percepción sobre el significado de la Educación Especial}

Para una mayor comprensión en cuanto a la interacción maestro- estudiante se plantea qué se entiende como Educación Especial. Para una adecuada atención de un 


\section{EDUCACIÓN}

programa educativo específico se requiere del docente que conozca de qué se trata, cuáles son sus propósitos, de lo contrario no podrá aportar al logro de los mismos de manera consciente y eficaz.

Con respecto a lo anterior, se indagó con relación a la percepción sobre la Educación Especial y se encontró que la subdirectora concibe que la "Educación Especial” es para atender a los niños con diferentes necesidades educativas, cuando su aprendizaje es muy lento o tienen mentalidad de infante". Lo mismo expresó la docente.

El Ministerio de Educación de Nicaragua (MINED: 2010) ha tomado la firme decisión de desarrollar una "Educación Inclusiva", fortaleciéndose el carácter de transversalidad de los servicios de Educación Especial, en los diferentes programas educativos.

Así mismo, el MINED plantea que se fortalece la atención a las Necesidades Educativas Especiales (N.E.E.) no asociadas a discapacidad, siendo coherentes con el enfoque de educación inclusiva, la cual indispensablemente requiere asumir la atención a la diversidad y recalca: "no se trata sólo de integrar al que estaba excluido sino de diseñar una educación que de entrada incluya a la diferencia".

Cabe señalar que para lograr el aprendizaje de una segunda lengua en niñas y niños con capacidades diferentes se requiere de mucho apoyo, atención especial, y por toda esta situación algunos autores confirman que un niño o niña con discapacidad física o mental no alcanzan o es imposible que alcancen esos niveles de educación desde una escuela regular.

En la actualidad la escuela "Hna. Maureen Courtney" es la única especial en la región que atiende a la niñez con este tipo de problemas. Los docentes que tienen a su cargo la educación en esta escuela, a pesar de que son docentes empíricos en esta modalidad, han logrado sacar adelante con sus propios esfuerzos y conocimientos a esos niños y niñas, utilizando estrategias, metodologías, atención especial e individual a cada uno de los estudiantes, según sus necesidades especiales.

Expresó la docente: "Nosotros como docentes tenemos que estar conscientes de que los niños especiales tienen el mismo derecho y oportunidades que otros". Podría afirmarse que tanto la subdirectora y docente están claras de la educación que debe brindar el centro y el papel que les corresponde desempeñar.

A pesar de no ser especialistas en su campo de trabajo, han puesto su empeño y dedicación para atender a la niñez, brindando algo muy importante como es el aspecto afectivo. 


\section{Métodos y medios didácticos utilizados}

\section{Métodos utilizados en la enseñanza aprendizaje del español}

Conforme el primer objetivo propuesto encontramos elementos importantes sobre los métodos utilizados en el desarrollo de las clases. La docente expresó que ella utiliza el método silábico, el Fonético-Analítico-Sintético (FAS) y el mixto, en el desarrollo de clases que imparte a estudiantes de primer grado en la asignatura de español. Sobre este mismo aspecto, la subdirectora manifestó que la docente debe emplear el método actual que es el activo e integrado.

En este aspecto se encontró un poco de contradicción entre lo expresado por la docente y por la subdirectora sobre el método implementado. En las observaciones se verificó que el método y organización del aula de la docente fue conforme una metodología tradicional. En el desarrollo de las clases se constató la falta de motivación al aprendizaje conforme las temáticas desarrolladas.

Para Arredondo (1989: 5,6), los métodos de enseñanza descansan sobre las teorías del proceso de aprendizaje y una de las grandes tareas de la pedagogía moderna ha sido estudiar de manera experimental la eficacia de dichos métodos, al mismo tiempo que intenta su formulación teórica.

En este campo sobresale la teoría psicológica: la base fundamental de todo proceso educativo se halla representado por un reflejo condicionado, es decir por la relación asociada que existe entre la respuesta y el estímulo que la provoca.

Se obvió el conocimiento y las experiencias propias de cada estudiante, lo que no se aprovechó para favorecer el enriquecimiento entre ellos. En este sentido Galdames V. (43,-48), afirma que uno de postulados de esta metodología es que: "los niños construyen sus aprendizajes participando activamente en situaciones auténticas y significativas".

Además plantea que las metodologías tradicionales, todavía están generalizadas en nuestros salones de clases, enfatizan los procesos de enseñanza utilizadas por el profesor, más que en los procesos de aprendizaje de los estudiantes.

El sistema educativo del centro de educación es monolingüe, pero la docente se auxiliaba con la lengua materna cuando era necesario para cumplir los objetivos propuesto. Los estudiantes expresaron que a ellos les gustaría recibir su clase en su lengua materna o bilingüe, ya que de esa manera asimilan y aprenden de mejor manera la segunda lengua. 


\section{EDUCACIÓN}

Al respecto, Venecia P. (2003), afirma que cuando se desconocen los pasos para la enseñanza- aprendizaje de la L2, los docentes pretenden que la niñez aprenda castellano a partir del uso que hacen de esta lengua para así enseñar contenidos en diferentes asignaturas o cualquier otro contenido. De hecho algo aprenderían, pero estarían lejos de lograr los objetivos en la adquisición en otra lengua como en su aprendizaje.

Sobre este mismo aspecto, Galdames V. (2008: 48), recalca la importancia de que los aprendizajes se den en situaciones auténticas y significativas para los niños, es decir que los contenidos y prácticas que se realizan en la sala de clase tengan relación lo más directa posible con la vida cotidiana de los estudiantes. Ello permite una comprensión más profunda de los contenidos, junto con la integración de las experiencias y habilidades previas.

Agrega el autor que estos aspectos se ven cualitativamente fortalecidos con el uso de materiales educativos, los que permiten desarrollar actividades grupales que resultan particularmente atractivas, puesto que integran el juego, una de las actividades que mejor caracterizan los intereses de la niñez.

Al finalizar el desarrollo de clase tampoco se evaluó el contenido de manera oral ni escrita. La docente obvió la evaluación que es un instrumento importante para comprobar el nivel de conocimiento y comprensión del estudiante sobre el tema desarrollado para que la calidad de educativa sea significativa.

Jarquín Chow, S. (2005: 10), afirma que para iniciar la enseñanza del español como segunda lengua, el docente debe tomar en cuenta el dominio que tienen los estudiantes a nivel oral de ese idioma. $\mathrm{Al}$ respecto, la consultora Fantini Beatriz C. (2001) afirma que el aprendizaje de una segunda lengua se lleva a cabo de una forma similar a la adquisición de la primera.

Es decir, que sigue un proceso o secuencia que se va desarrollando por etapas. De esta manera, la soltura o fluidez va aumentando en una forma determinada y la organización del lenguaje va de lo más simple a lo más complejo: pre-producción, producción preliminar, producción de oraciones completas, producción intermedia y avanzada.

Complementan esta idea Alfonso E. y Jeldres M. (1,999), quienes señalan que las competencias de comprensión y producción se logran a través del desarrollo de las cuatro habilidades lingüísticas a saber: hablar, escuchar, leer y escribir.

Podría afirmarse que la metodología para la enseñanza de la L2 está aún muy empírica en cuanto a su aplicación para estudiantes de primer grado de Educación Especial. Según observaciones realizadas, la metodología utilizada para enseñar L2 es muy diferente a la utilizada para enseñar L1, lo que provoca problemas de aprendizaje 
en la L2. Durante las observaciones realizadas en el aula de clase con relación a la metodología implementada por la docente se constata que se desarrolla en forma monolingüe.

Algunos estudiantes expresaron que tienen problemas en la asimilación y comprensión del español por falta de conocimiento de algunos sonidos de la misma, por eso expresó uno de ellos: "me gustaría que el desarrollo de la clase fuera en dos idiomas y así voy a poder asimilar, comprender y alcanzar con éxito el objeto propuesto".

También la docente nos informó que los estudiantes tienen muchas dificultades en aprender el lenguaje español debido a que muchos proceden de comunidades aledañas y su lengua materna es el miskitu. Además, la docente considera que es necesario emplear una metodología adecuada para la enseñanza de la L2, para la etnia miskitu, pues de esta manera elevaría la pro eficiencia lingüística en el aula de clase para enseñar el español como segunda lengua.

Para todo docente el método es la base fundamental de la enseñanza de una segunda lengua. Se necesita que la docente deba utilizar un método más apropiado para que el estudiantado pueda asimilar bien lo que va aprender. Repetir varias veces una palabra con buena pronunciación y una entonación clara hasta que los estudiantes aprendan bien a pronunciar, leer y escribir, y así para el logro de su aprendizaje de la segunda lengua.

Para Venecia el aprendizaje o adquisición de una segunda lengua debe realizarse a través de prácticas pedagógicas apropiadas con el apoyo técnico educativo, y sobre todo con la guía de docentes que manejen métodos adecuados para su enseñanzaadquisición.

La autora Carmen Muñoz, afirma que la diferencia que existe entre la lengua materna y una segunda lengua, es que la materna la vienen hablando dentro del entorno familiar en que se comunican en una comunidad determinada. Mientras que la segunda lengua es la que uno aprende en la escuela y nuestra relación social con otras personas del habla española. Además, afirma que los términos lengua materna y una segunda lengua se diferencian entre sí; en el primer caso, se trata de una lengua hablada en la comunidad en que vive, mientras en el segundo caso la lengua no tiene presencia en la comunidad.

La consultora Fantini expresa que el aprendizaje de una segunda lengua se lleva a cabo de una forma similar a la adquisición de la primera, por medio de un proceso que se desarrolla por etapas: pre-producción, producción preliminar, producción de oraciones completa, producción intermedia y avanzada. Para lograr con éxito este objetivo se necesita emplear métodos adecuados para la enseñanza aprendizaje. Los 
planteamientos de Venecia Mauceri, P. Carmen Muñoz y la consultora Fantini son similares por que los tres planteamientos tiene una relación lógica entre sí.

En la presentación, según Jarquín Chow, S. (5-10) el o la docente utiliza diversos recursos para que los estudiantes entiendan lo que dicen en español: gestos, mímicas, láminas, objetos, títeres, y sobre todo una pronunciación clara y una entonación natural adecuada.

En la práctica recomendamos las siguientes técnicas para ser empleadas sucesivamente:

- Repetición.

- Diálogo - docente- estudiante.

- Diálogo entre estudiantes.

Plantea que el objetivo es que los estudiantes usen el español para otras actividades, dentro y fuera de la escuela. Los juegos y las canciones son buenos recursos para iniciar el uso de la segunda lengua, aun cuando sea poco el vocabulario y limitado el número de estructura que los estudiantes manejan.

\section{Medios didácticos}

Necesariamente, si la docente desea que una metodología de enseñanza sea exitosa, debe estar acompañada de algún material didáctico. Los materiales didácticos son todo objeto que se puede manipular manualmente para la enseñanza. Estos pueden ser artificiales y naturales en el proceso de enseñanza.

También se conocen como un medio de apoyo, práctico y complementario para enriquecer más nuestros métodos de enseñanza. Además es un instrumento importante motivador en la enseñanza siempre y cuando sea adecuado y significativo para los estudiantes en relación con el contenido desarrollado.

De acuerdo con lo manifestado por la docente respecto a los medios didácticos, expresó que utiliza carteles, recortes de papeles y que el centro les facilita suficiente material didáctico para la elaboración de materiales en trabajos para su desarrollo de clase. Al respecto, la subdirectora manifestó que en primer grado, lo más que utilizan son láminas, libros de textos, figuras atractivas, chalupas y el rincón de aprendizaje.

Con las observaciones realizadas se constató que durante el desarrollo de clase la docente no utilizó ningún tipo de material elaborado para mejorar la calidad de la enseñanza del español. Esto contradice lo que la docente plantea con relación a que en la escuela se les provee del material necesario para el desarrollo de las clases. 
Al no utilizar medios y materiales didácticos se pierde información y motivación. En este sentido Raymond (1978: 7) afirma que los materiales didácticos utilizados proporcionan información práctica, fácilmente comprensible e instrucciones de tipo manual sobre fotografía, elaboración de diapositivas, rótulos, dibujos, tableros de anuncios, montaje de carteles en seco y de muchos otros recursos del oficio.

Estos aspectos se ven cualitativamente fortalecidos con el uso de materiales educativos, los que permiten desarrollar actividades grupales que resultan particularmente atractivas, puesto que integran el juego. El juego es una de las actividades que mejor caracteriza los intereses de la niñez.

Además, recomienda presentar la información de modo ilustrativo, para alentar al principiante a iniciar el trabajo bajo su responsabilidad. Esto deja implícito que no basta con tener el material para la enseñanza, lo más importante es cómo este es presentado a los estudiantes y sobre todo cómo se utiliza.

\section{Interacción maestro - estudiante en el proceso de educativo}

La interacción maestro - estudiante es el grado de comunicación, de entendimiento, respeto y afecto que se da entre ellos. Montaner (1993: 21) define que la Comunicación Pedagógica, permite establecer relaciones con el medio ambiente, con los demás y consigo mismo. En el ámbito humano, existen diversas maneras de relación del cual surgen diferentes significados en torno al término "Comunicación".

Recalca que de esas distintas relaciones interesa una en particular, la relación maestro-estudiante, porque la comunicación, no es nada ajeno al proceso educativo, si no que están íntimamente ligados, no se puede concebir una sin el otro. En la observación de clase se pudo constatar que hay muy buena relación entre la docente y los estudiantes. Se percibió un ambiente adecuado para una buena comunicación, respeto y confianza mutua entre ellos.

La buena comunicación es una de las actividades más importantes de las que se producen a lo largo del día y permite el buen funcionamiento de las sociedades humanas, las personas participamos interrumpidamente en actos de comunicación desde que el día se inicia hasta que termina.

La buena comunicación que mantiene la docente con el estudiantado es fundamental para mejorar la buena marcha en la enseñanza aprendizaje, lo cual es primordial en todo proceso educativo. La confianza mutua observada es fundamental en el proceso de aprendizaje, facilita la ruptura de cualquier tipo de barreras para el aprendizaje de una segunda lengua. 
Elkorin (1990: 292) dice que la función del maestro es la presentación del proceso pedagógico, partimos de la importancia de la actividad del educador. El educador guía el proceso pedagógico, planifica y dirige la actividad de sus estudiantes de manera que se logre la influencia educativa prevista. Se puede asegurar que el papel dirigente del maestro en su proceso docente está objetivamente condicionado. Es el resultado de su encargo social, del papel social de su profesión y consiste en la transmisión a la nueva generación de la riqueza cultural acumulada por la humanidad. Conforme lo observado puede afirmarse que en la interacción docente - estudiantes hay excelente comunicación, confianza y respeto que facilitan el proceso enseñanza - aprendizaje.

\section{Función del estudiante}

Los estudiantes tienen un sin número de tareas a cumplir en un aula de clase, para que reciban una educación integral. Están obligados a mantener el orden, aseo, disciplina y participar de manera activa en todas las materias que el docente imparte. Durante las observaciones realizadas en el aula de clase de primer grado, en relación a la función del estudiante, se observó que estaban muy atentos en la clase, participaban activamente, hacían preguntas en relación al contenido, intercambiaban sus ideas y sentimientos sobre los conceptos básicos que le ayudan a su formación integral.

Durante la observación de clase, el docente realizó un papel importante en cuanto a la atención del aprendizaje de los estudiantes especiales. La interrelación maestroestudiante se dio de manera directa e integral en el proceso de la enseñanza aprendizaje. Durante el desarrollo del contenido aplicó una adecuada presentación, desde el inicio de las actividades, demostrando sus habilidades y destrezas con una técnica especializada teórica y práctica para su entorno en el ámbito estudiantil dentro del aula.

\section{Conclusiones}

Se percibe la Educación Especial como aquella en la que debe brindarse atención diferenciada e individual a cada uno de los estudiantes según sus necesidades especiales con elementos fundamentales como: afecto, cariño, amor, confianza mutua, comunicación, valoración y tratamiento como a cualquier otro niño o niña, que tiene el derecho de ser parte integral y activa de la sociedad.

El conocimiento y dominio de la metodología para la enseñanza del español por parte de la docente, es aún muy empírica en cuanto a su aplicación hacia los estudiantes de primer grado de Educación Especial.

Para el uso adecuado del método de enseñanza del español se necesita que la docente utilice mejores estrategias para que los estudiantes puedan asimilar correctamente lo que se va a aprender. 
A pesar de la existencia de medios didácticos para la enseñanza del español, son poco utilizados, lo que trae como consecuencia la pérdida de información y correcta motivación para aprender una lengua que no es la materna.

Hay una excelente interacción maestro-estudiante basada en la confianza y el respeto en el desarrollo del proceso educativo del español, pero no es aprovechada adecuadamente por falta de dominio de las metodologías del español.

La interacción maestro-estudiante favorece la realización de actividades creativas y recreativas de aprendizaje.

\section{Lista de referencias}

Alfonso E. \& Jeldres. M. (1999). Decodificación Sintáctica en Español Hablado. Un Antecedente Comprensión Auditiva en Lengua Extranjera. Revista Signos, V. XXIV, 2 sem. Pág. 40.

Arredondo, M. (1989). Notas para un modelo de docencia formación pedagógica de profesores universitarios. Teoría y experiencias de México. México: ANUIES-UNAM. CESU. Pág. 5,6.

Babanski, Yu (1990) Pedagogía. Editorial de libros para la Educación Pág. ,287.

Elkorin 1990 Pedagogía editorial de libros para la educación. Pág. 292.

Guillén, C. y Castro, P. (1998) Manal de Autoformación para una didáctica de la lengua cultura extranjera. Madrid, La Muralla. Pág. 95.

Galdames, V. (2008). Pontificia Universidad Católica de Chile. Dirección de educación a distancia material educativos en la sala de clase. Biblioteca de aula y materiales de lengua y matemática. Pág. 43,48.

Jarquín Chow, Selmira. (2008) Bases filosóficas de la Escuela de Educación Especial Hna. "Maureen Courtney", p. 2.

Montaner Pedro (1993). ¿Cómo nos comunicamos? 2da. Edición, Al hombre mexicano, Pág. 21.

Muñoz Carmen (2002). Aprender idioma. Barcelona, Ediciones Paidós S. A. Pág. 112 -113

Pérez Gómez, A. (1992). La función y formación del profesor en la enseñanza para la comprensión: comprender y transformar la enseñanza, Madrid, Ediciones Morata. 
Raymond V. Wiman, (1978) Material Didáctico, Pág. 7,8.

Regueira A, Guadamuz de M. (1987). Documentación Bibliografía, Administración escolar, Pág. 4.

Venecia Mauceri, Paolo, (2003) Educación Intercultural y Plurilingue. Primera edición Serie Materiales de apoyo para la formación docente en Educación Intercultural. Managua Terra Nuova, pp. 139-141.

Medellín V, Lorely, (2006). Interacción Maestro alumno. http//uva. analhuac. mx/ mace/foro/modulo7. 1/224/ltml

Morin Edgar (2009): Definición de Educación especial. http: //definición. de/educaciónespecial/. Pág. 2,3.

Ministerio de Educación. (2010) Educación especial. Sitio web: www. mined. gob. ni. [Visitado en enero 2010].

Ministerio de Educación. (2006) Necesidades educativas especiales en Nicaragua. Sitio web. www. mined. gob. ni 